\title{
ANÁLISIS CIENTIMÉTRICO DE LAS \\ PUBLICACIONES INDEXADAS EN JOURNAL CITATION REPORTS SOBRE EDUCACIÓN FÍSICA
}

\author{
ANÁLISE CIENTOMÉTRICA DE PUBLICAÇÕES INDEXADAS NO JOURNAL \\ CITATION REPORTS SOBRE EDUCAÇÃO FÍSICA
}
SCIENTOMETRIC ANALYSIS OF PUBLICATIONS INDEXED IN JOURNAL CITATION REPORTS ON PHYSICAL EDUCATION

\author{
Francisco-Javier Hinojo-Lucena*, Inmaculada Aznar-Díaz*, María-Pilar \\ Cáceres-Reche*, José-María Romero-Rodríguez*
}

\section{Palabras clave:}

Educación Física.

Publicaciones

periódicas.

Bibliometría.

Factor de impacto.
Resumen: En los últimos años, la Educación Física se ha consolidado como una disciplina de estudio que acapara gran interés. Como consecuencia, la producción científica ha ido en aumento, reflejándose en la cantidad de artículos que se publican sobre el tema. El objetivo de este trabajo ha sido analizar la producción científica sobre Educación Física, indexada en Web of Science, desde sus inicios hasta el año 2017. Para ello, se ha realizado un estudio bibliométrico, recogiendo los indicadores de producción, dispersión e impacto de la producción científica en la Educación Física. Entre los resultados, se constata que la literatura se encuentra en una etapa de crecimiento exponencial. Además, desde los inicios de la producción científica, en 1902, sobre este tópico se han configurado determinadas revistas, instituciones, países y autores como referentes en la temática. Finalmente, el estudio ofrece una visión general del avance de las publicaciones de impacto sobre Educación Física.

Resumo: Nos últimos anos, a Educação Física se estabeleceu como um campo de estudo de grande interesse. Como consequência, a produção científica tem vindo a aumentar, o que se reflete na quantidade de artigos publicados sobre o assunto. 0 objetivo deste trabalho foi analisar a produção científica em Educação Física, indexada em Web of Science, desde o seu início até 2017. Para isso, foi realizado um estudo bibliométrico, reunindo os indicadores de produção, dispersão e impacto da produção científica na Educação Física. Dentre os resultados, observa-se que a literatura está em fase de crescimento exponencial. Além disso, desde o início da produção científica sobre esse tema, em 1902, alguns periódicos, instituições, países e autores foram configurados como referências no tema. Finalmente, o estudo fornece uma visão geral do progresso das publicações de impacto sobre Educação Física.

Abstract: In recent years, Physical Education has established itself as a field of study

Keywords Physical Education. Journals. Bibliometrics. Impact factor. of great interest. Consequently, scientific production has been on the rise, reflected in the number of publications on the topic. This article analyzed scientific production on Physical Education indexed in Web of Science, from its beginnings until 2017. For this, a bibliometric study collected the indicators of production, dispersion and impact of the scientific production on Physical Education. Among the results, it found that the literature is in a stage of exponential growth. Furthermore, since scientific production on the topic began in 1902, certain journals, institutions, countries and authors have been established as benchmarks on the topic. Finally, the study reflects a general overview on the progress of impact publications on Physical Education.
*Universidad de Granada. Granada España.

E-mail: fhinojo@ugr.es;

iaznar@ugr.es;

caceres@ugr.es;

romejo@ugr.es

Recebido em: 19-11-2018

Aprovado em: 23-04-2019

Publicado em: 17-05-2019

DOI

https://doi.org/10.22456/1982-8918.88273

(c) (i) () Licence 


\section{INTRODUCCIÓN}

En los últimos años las investigaciones sobre educación física se han incrementado, la introducción de esta materia en la Universidad ha hecho que surja una línea de investigación centrada en este tópico (VALENCIANO; DEVÍS; BELTRÁN, 2008). Del mismo modo, la producción científica ha ido en aumento, lo cual se refleja en la cuantía de revistas que publican artículos relacionados con la educación física.

En este escenario, la investigación debe ir aparejada obligatoriamente de la comunicación de los hallazgos, puesto que carecen de relevancia si no se comunican. Así pues, la publicación en formato de artículos es un medio esencial para poder transferir los resultados de investigación (REVERTER; HERNÁNDEZ; JOVÉ; FONSECA; LEGAZ, 2014). No obstante, es fundamental discernir entre las publicaciones que se realizan. En función de la tipología de la revista podrían estar introduciéndose artículos que carezcan de rigor científico y por tanto, los datos no sean relevantes.

Para paliar esta problemática surge el factor de impacto de las revistas, destacando entre todos ellos el Journal Citation Reports (JCR). Este índice proporciona una metodología cuantitativa de evaluación de revistas científicas, cuya indexación en la base de datos Web Of Science (WOS) y la obtención del índice de impacto JCR dota de rigurosidad el artículo científico y actúa como indicador de calidad de la publicación (BUELA-CASAL; ZYCH, 2010; HERNÁNDEZ; REVERTER; JOVÉ; MAYOLAS, 2013).

Centrándonos en el tópico del trabajo, el concepto de educación física se define como "una acción educativa que atañe a toda la persona, no sólo a su cuerpo" (LÓPEZ, 2006, p. 19). Por lo que entra en juego el desarrollo de diferentes capacidades tanto físicas como sociales y mentales. Asimismo, la principal implicación que presenta la educación física en los estudiantes es, el aprender a convivir, a partir de la aceptación de reglas, respeto a la autonomía personal, la participación y la atención a la diversidad (HINOJO-LUCENA; AZNAR; CÁCERES, 2016)

Por otro lado, entre los beneficios que proporciona la actividad física, se sitúa un mayor bienestar psicológico, el aumento del autoconcepto y una menor percepción del estrés (GARCÍA, 2018; MANZANO; LÓPEZ; SUÁREZ; RUIZ, 2018; MORAL, 2018; RAMÍREZ; RAYA; RUIZ, 2018).

Atendiendo a estudios bibliométricos previos en esta línea, cabe resaltar su focalización en distintos aspectos:

a) Reverter y Munguía (2007), se centraron en el estudio de tres revistas de educación física (Apunts, Revista de Psicología del Deporte y Revista Tándem), donde se analizaron los índices correspondientes al total de citas nacionales e internacionales, cuantía de trabajos publicados y el factor de impacto.

b) Villamón, Job, Valcárcel y Devis-Devis (2012), desarrollaron un análisis comparativo de cinco revistas del campo de la educación física/ciencias del deporte a partir del establecimiento de 15 indicadores de transparencia, gestión editorial, visibilización e internacionalización. Las revistas analizadas fueron: Motriz. Revista de Educação Física, Movimento, Revista Brasileira de Medicina do Esporte (RMBE), Revista de Psicología del Deporte y Revista Internacional de Medicina y Ciencias de la actividad Física y el Deporte (RIMCAFD). 
c) Reverter, Hernández, Jové y Legaz (2013), llevaron a cabo una investigación sobre los hábitos de publicación de los profesores de Educación Física españoles en WOS, analizando distintos indicadores de productividad: revistas, instituciones y autores con mayor cuantía de documentos.

d) Reverter et al. (2014), realizaron una comparativa entre las publicaciones del profesorado universitario de educación física en España y Brasil. Las revistas indexadas en JCR donde más publican los docentes españoles son Journal of Strengh and Conditioning Research (JSCR) y Revista de Psicología del Deporte, mientras que los docentes brasileños registran una mayor cuantía de documentos en Medicine and Science in Sports y en JSCR.

e) Olivera y Andrés (2017), hicieron un análisis de la revista Apunts. Educación Física y Deportes durante el periodo de 1985-2015. Se recogen datos referentes a su productividad diacrónica, categorías temáticas, autoría, países e indexación.

f) Santos, Lima, Cassani y Ferreira (2018), se centraron en artículos de revistas que trataban el tópico sobre evaluación de la educación física escolar publicados desde 1932 hasta 2014. Se analiza la producción por años, revistas y autores. La producción se encuentra en periodo de crecimiento exponencial.

g) Rubilar y Pérez (2018), analizaron los artículos publicados en revistas chilenas de ciencias del deporte durante el gobierno militar. Atienden las variables correspondientes al año de publicación, disciplina científica, instituciones, autores y países.

h) Gomes, Quintao, Marinotte, Sampaio y Rossini (2018), recogieron los datos referentes a los artículos en cinco revistas de educación física brasileñas: Motrivivência, Motus Corporis, Pensar a Prática, Revista Brasileira de Ciência e Movimento (RBCM) y Revista da Educação Física (UEM). Analizan las variables: año de publicación, revistas, temáticas, tipología de los textos, autoría, titulación y filiación institucional y regional.

A partir de la revisión de estos estudios, surge la necesidad de abordar de forma general el tópico principal "educación física". Los análisis bibiliométricos previos indagan aspectos específicos dentro de la problemática, centrando su atención en particularidades y no en el conjunto. Por tanto, en este trabajo se propuso como objetivo analizar la producción científica sobre educación física, indexada en WOS, desde sus inicios hasta el año 2017.

Los interrogantes que guiaron el proceso de investigación fueron:

- ¿Cuál es el estado de la producción científica sobre educación física a lo largo del tiempo?

- ¿Existe una relación productiva entre autores y el número de artículos publicados?

- ¿Se concentra la mayor parte de artículos en un número reducido de revistas?

- ¿Cuáles son las principales revistas, instituciones, autores y países con mayor producción científica sobre educación física?

\section{METODOLOGÍA}

Este estudio sigue la metodología propia que caracteriza a un análisis bibliométrico (GLANZEL; SCHOEPFLIN, 1999). Con la finalidad de abarcar el objetivo del estudio, se aplicó como único descriptor el término "Physical Education". Este descriptor es aceptado 
comúnmente por la comunidad científica, hallándose en el tesauro de la UNESCO y en el tesauro de Education Resources Information Center (ERIC). En el proceso de búsqueda se utilizó la opción "búsqueda básica" y se introdujo el descriptor "Physical Education", seleccionando la categoría "topic" para no limitar los resultados iniciales. La búsqueda en la base de datos WOS se realiza en idioma inglés, por lo que el término fue introducido en esta lengua. De modo que se recoge la producción científica desde su origen en 1902 hasta el año 2017. Cabe resaltar que la búsqueda tuvo lugar durante el último trimestre del año 2018.

\subsection{MUESTRA}

La unidad de análisis se ha compuesto por los artículos de revista indexados en WOS sobre el tópico educación física $(n=7.939$ ). La muestra final se extrajo del cómputo total de producción científica de WOS a partir de la aplicación de los criterios de inclusión y exclusión (tabla 1).

Tabla 1 - Criterios de inclusión y exclusión.

\begin{tabular}{ll}
\hline Criterios de inclusión & Criterios de exclusión \\
\hline (a) Artículos de revista. & (a) Actas de congresos, capítulos de libro, libros u otro tipo de \\
(b) Indexación en los índices de WOS: Social & publicaciones. \\
Sciences Citation Index (SSCI); Science & (b) Indexación en los índices de WOS: Emerging Sources \\
Citation Index Expanded (SCIE) y Arts and & Citation Index (ESCI); Conference Proceeding Citation Index \\
Humanities Citation Index (AHCI). & (CPCI) y Book Citation Index (BCI). \\
\hline
\end{tabular}

Fuente: elaboración propia.

El diagrama de flujo muestra el proceso de refinado para la obtención de la muestra definitiva (figura 1).

Figura 1 - Diagrama de flujo.

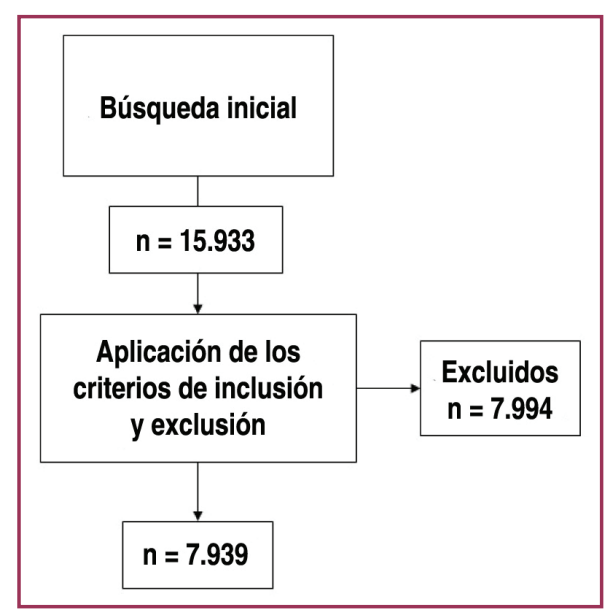

Fuente: elaboración propia.

\subsection{ANÁLISIS DE DATOS}

Las variables de análisis se han establecido en base a estudios bibliométricos previos (REVERTER; MUNGUÍA, 2007; REVERTER et al., 2013; OLIVERA; ANDRÉS, 2017; GOMES et al., 2018; RUBILAR; PÉREZ, 2018; SANTOS et al., 2018) y a la aplicación de las leyes de la 
bibliometría (PRICE, 1986; GONZÁLEZ; MOYA; MATEOS, 1997; ROUSSEAU; ROUSSEAU, 2000; GUTIÉRREZ; MARTÍN; CASASEMPERE; FERNÁNDEZ, 2015; URBIZAGASTEGUI, 2016). Asimismo, se establecieron los siguientes indicadores bibliométricos:

- Indicadores de producción: productividad diacrónica (verificación de la ley de Price) y productividad de autores (confirmación de la ley de Lotka).

- Indicadores de dispersión: comprobación de la ley de Bradford.

- Indicadores de impacto: productividad de revistas, instituciones, autores y países.

El análisis de datos se ha realizado a partir de la información obtenida en WOS. Además, algunos análisis han requerido del uso de software específico: Excel para las correlaciones entre autores-número de artículos y revistas-número de artículos y UCINET para establecer la colaboración entre autores.

\subsection{LIMITACIONES}

Una de las limitaciones que presentan los estudios bibliométricos se vincula con el motor de búsqueda. Por tanto, aquellos artículos que no recojan en el título, resumen o palabras clave el descriptor "Physical Education" pueden ser excluidos de los resultados finales. Sin embargo, la cuantía de documentos que se pueden analizar a partir del análisis bibliométrico permite obtener datos valiosos que representan una visión real del estado actual del tópico de búsqueda.

\section{RESULTADOS}

Los resultados se han dividido en función del indicador al que pertenecen. En síntesis, los datos obtenidos muestran la situación actual de la producción científica sobre educación física.

\subsection{INDICADORES DE PRODUCCIÓN}

La productividad diacrónica refleja la evolución de las publicaciones a lo largo del tiempo (figura 2). Si nos fijamos en los datos, el inicio de las publicaciones sobre educación física tuvo lugar en el año 1902. A partir de esa fecha la producción ha ido creciendo, excepto en los años 1909, 1913-1916, 1919 y 1929 donde se recogen cero publicaciones. El aumento notable de las publicaciones se experimentó en la década de los 90 hasta hoy, recogiendo el máximo de documentos en 2017 con 675 publicaciones (8,50\%).

La ley de Price establece que pasados 10 años la literatura científica tiende a duplicarse y se diferencian tres etapas en la producción científica: precursores, crecimiento exponencial y crecimiento lineal (PRICE, 1986). Para verificar esta ley bibliométrica se ha organizado la producción en quinquenios (GUTIÉRREZ et al., 2015). Se observa que esta premisa no se cumple regularmente, puesto que en los periodos de 1902-1912, 1933-1943, 1943-1953, 19531963 y 1973-1983 la producción no tiende a duplicarse. Sin embargo, en el resto de periodos temporales si se duplica. Los más significativos los encontramos en 1983 (12 documentos: ,15\%) a 1993 (58 documentos: ,73\%) y de 2003 (170 documentos: 2,14\%) a 2013 (525 documentos: 6,61\%). 
En cuanto a la etapa en la que se sitúa la producción científica, se distinguen dos de las tres etapas de las que habla Price. La etapa de precursores en el periodo que abarca desde 1902-1987 y a partir del año 1988 hasta 2017 la literatura se encuentra en una etapa de crecimiento exponencial. De modo que sigue actualmente en pleno desarrollo.

Figura 2 - Productividad diacrónica organizada por quinquenios.

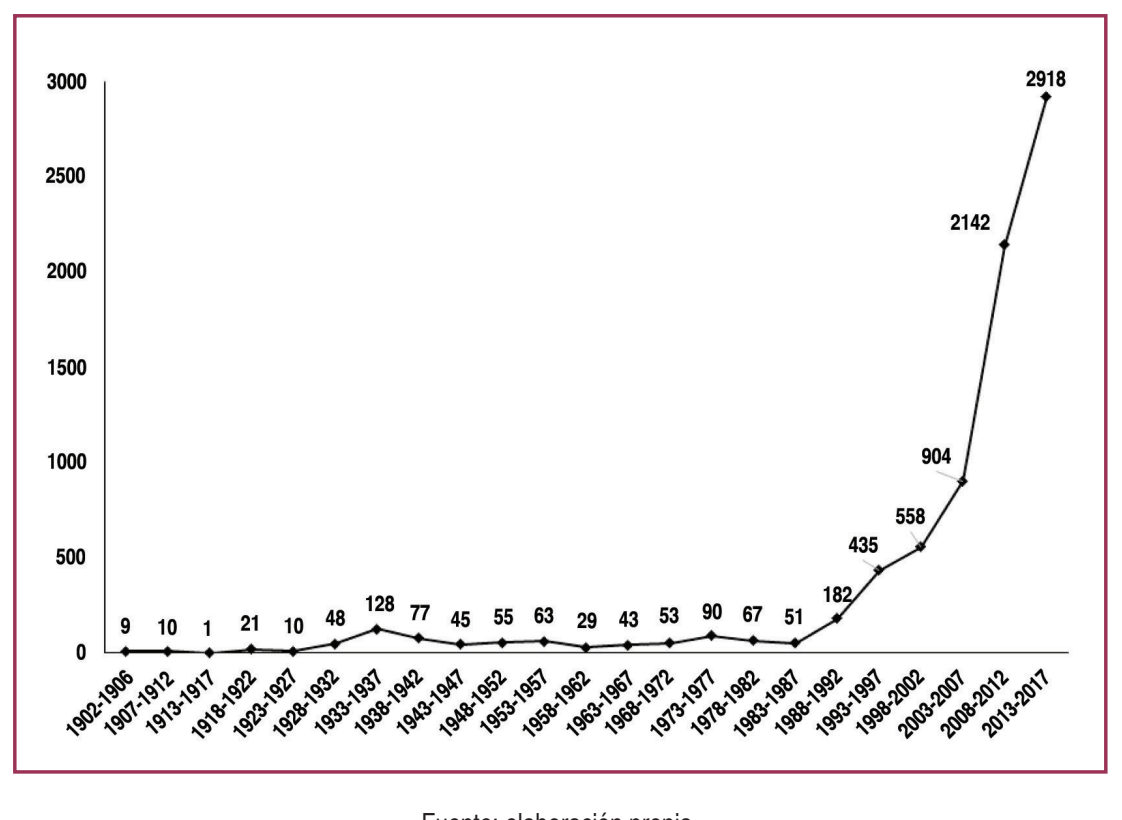

Por otro lado, la productividad de los autores se mide en función de la cuantía de documentos publicados. La ley bibliométrica de Lotka indica que un pequeño grupo de autores produce una gran cantidad de documentos, siendo estos autores muy productivos (ROUSSEAU; ROUSSEAU, 2000). Así sucede en la producción científica sobre educación física, donde una gran parte de artículos provienen de los mismos autores. En este sentido, ocho autores concentran más de 40 documentos cada uno.

En concreto, la producción científica de educación física se sustenta en 205 autores considerados grandes productores ( $>=10$ artículos) (6,89\%), 2.033 autores clasificados como medianos productores (>1<= 9 artículos) $(68,38 \%$ ) y 735 autores que son pequeños productores (<= 1 artículo) $(24,77 \%)$.

La regresión lineal evidencia lo establecido por la ley de Lotka, verificándose su principal premisa (figura 3). Se muestra la correlación negativa entre el número de autores y artículos ( $r$ $=-.55)$, ecuación del gráfico $\left(\mathrm{y}=133,18 \mathrm{e}^{-0,121 x}\right)$ y ajuste del modelo $\left(R^{2}=.77\right)$. 
Figura 3 - Correlación entre autores (eje Y) y artículos (eje X).

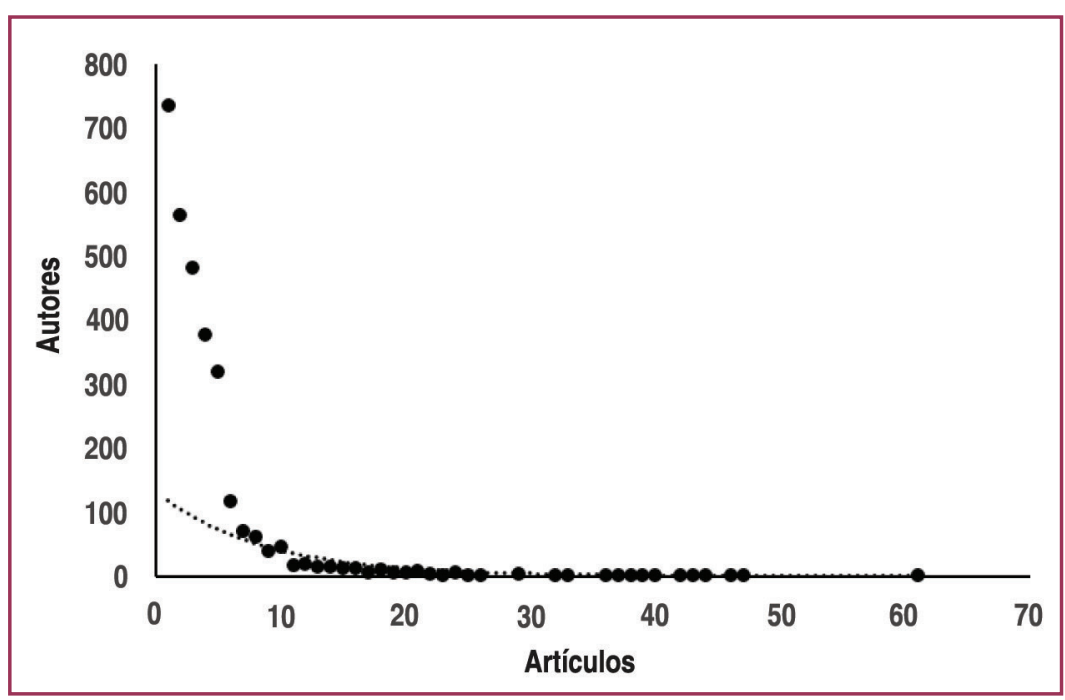

Fuente: elaboración propia a partir de Excel.

\subsection{INDICADORES DE DISPERSIÓN}

El cálculo de la dispersión de la producción científica atiende a cómo se distribuyen las revistas en base a la cuantía de documentos que publican sobre educación física. La ley de Bradford establece que si se realiza un reparto equitativo de artículos en distintas zonas, el núcleo presenta una cantidad equivalente a pesar de contener menor cuantía de revistas (URBIZAGASTEGUI, 2016). Al igual que la ley de Lotka, se indica que un pequeño grupo de revistas concentra una gran cantidad de artículos.

La aplicación de la ley de Bradford muestra la distribución en cinco zonas con la misma cantidad de artículos aproximadamente $(M=1.587)$. En este sentido, se observa que el núcleo conformado por cuatro revistas contiene una cantidad similar de documentos al resto de zonas (figura 4). El total es de 938 revistas y 7.939 artículos.

Figura 4 - Áreas de dispersión de Bradford.

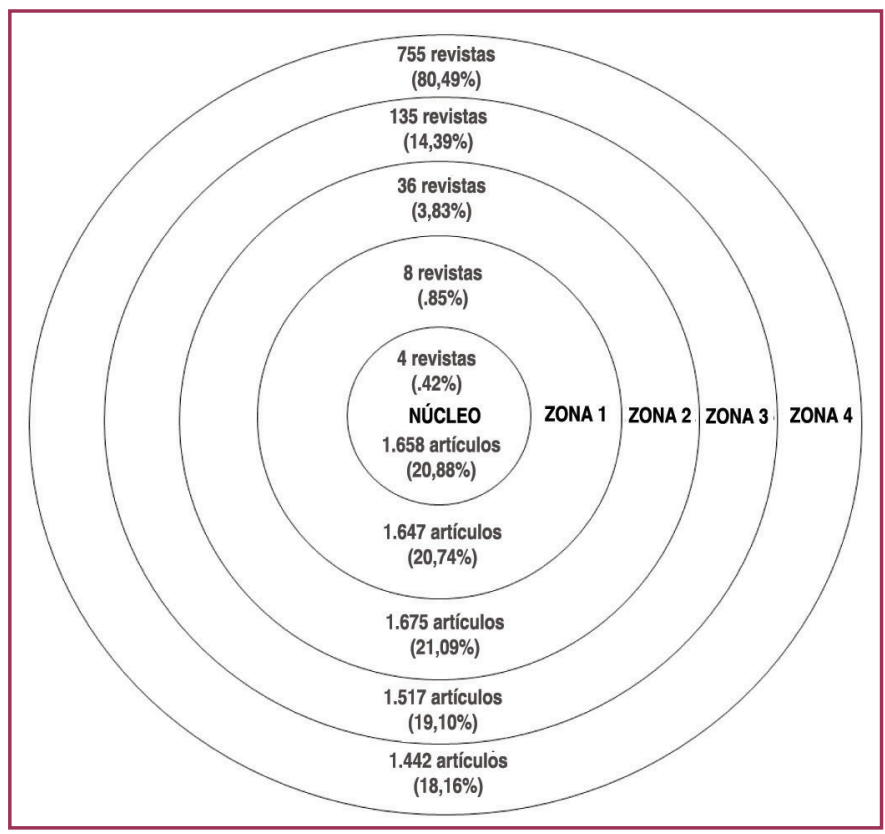

Fuente: elaboración propia. 
Complementariamente, se ha realizado una regresión lineal entre el número de revistas y artículos para constatar la premisa establecida en la ley de Bradford (figura 5). Así pues, se refleja la correlación negativa entre las dos variables $(r=-.17)$, ecuación del gráfico ( $\mathrm{y}=$ $\left.4,0412 \mathrm{e}^{-0,005 x}\right)$ y ajuste del modelo $\left(R^{2}=.17\right)$. Se afirma la tendencia acerca de que un pequeño clúster de revistas publica una gran cantidad de documentos sobre la temática educación física.

Figura 5 - Correlación entre revistas (eje Y) y artículos (eje X).

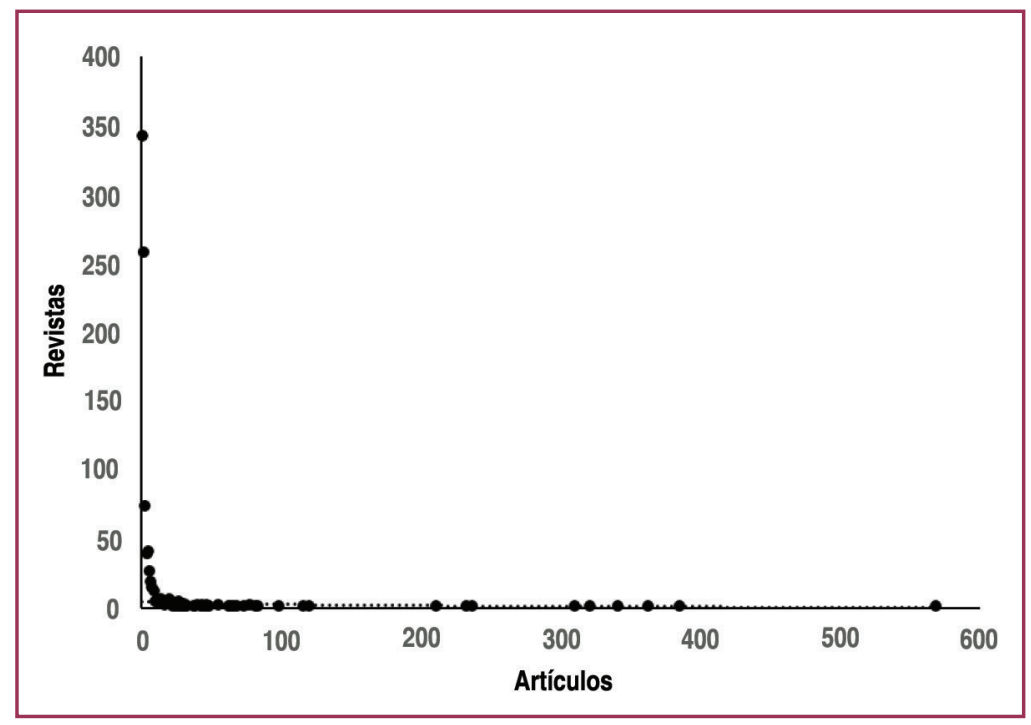

Fuente: elaboración propia a partir de Excel.

\subsection{INDICADORES DE IMPACTO}

En estos años se han ido posicionando como referentes distintas revistas, instituciones, países y autores en la temática sobre educación física. Destacando sobre el resto en base a la cuantía de documentos que presentan y su índice de impacto (citas/documentos). Para establecer las de mayor cuantía e impacto, se optó por analizar las 10 primeras revistas, instituciones, países y autores con más producción científica. Esta acotación en el cuantitativo de 10 se estableció con la finalidad de dar viabilidad al análisis en base al gran número de resultados obtenidos. No obstante, el top 10 es un elemento común de acotación en distintos estudios bibliométricos (GUTIÉRREZ et al., 2015; OLIVERA; ANDRÉS, 2017; RUBILAR; PÉREZ, 2018).

En relación a las revistas, las cuatro primeras conforman el núcleo de Bradford (JTPE, Sport, Education and Society, RQES y Movimento), el resto forma parte de la zona 1 (tabla 2). Resalta como aquella más productiva Journal of Teaching in Physical Education (JTPE) (569 documentos, 7,16\%) y la más impactante Research Quarterly for Exercise and Sport (RQES) (8.559 citas en 363 documentos). Por su parte, Sport, Education and Society presenta 385 documentos (4,84\%) y un total de 6.605 citas; Movimento 341 documentos $(4,29 \%)$ y 381 citas; Quest 321 documentos (4,04\%) y 5.185 citas; European Physical Education Review (EPER) 237 documentos (2,98\%) y 2.146 citas; Physical Education and Sport Pedagogy (PESP) 233 documentos (2,93\%) y 2.248 citas; Perceptual and Motor Skills (PMS) 211 documentos (2,65\%) y 1.726 citas; Journal of School Health (JSH) 120 documentos (1,51\%) y 3.179 citas; Motriz Revista de Educação Física 116 documentos (1,46\%) y 157 citas. 
Tabla 2 - Revistas con mayor producción.

\begin{tabular}{lcccc}
\hline Revista & Artículos & $\%$ & Citas & I $^{*}$ \\
\hline Journal of Teaching in Physical Education (JTPE) & 569 & 7,16 & 10.892 & 19,14 \\
Sport, Education and Society & 385 & 4,84 & 6.605 & 17,15 \\
Research Quarterly for Exercise and Sport (RQES) & 363 & 4,57 & 8.599 & 27,73 \\
Movimento & 341 & 4,29 & 381 & 1,11 \\
Quest & 321 & 4,04 & 5.185 & 16,15 \\
European Physical Education Review (EPER) & 237 & 2,98 & 2.146 & 9,05 \\
Physical Education and Sport Pedagogy (PESP) & 233 & 2,93 & 2.248 & 9,64 \\
Perceptual and Motor Skills (PMS) & 211 & 2,65 & 1.726 & 8,18 \\
Journal of School Health (JSH) & 120 & 1,51 & 3.179 & 26,49 \\
Motriz Revista de Educação Física & 116 & 1,46 & 157 & 1,35 \\
\hline
\end{tabular}

Fuente: datos de WOS. Nota: $I^{\star}=$ Índice de impacto.

Entre las instituciones de referencia en la temática, destaca la California State University (CSU) como mayor productora (192 documentos, 2,41\%) y la University of Texas System (UT System) como la que más impacto presenta (4.814 citas en 113 documentos). Por otro lado, la University of North Carolina (UNC) obtiene 162 documentos (2,04\%) y 4.517 citas; Ohio State University (OSU) 144 documentos (1,81\%) y 3.082 citas; Loughborough University (LBORO) 142 documentos (1,78\%) y 4.463 citas; University of Queensland (UQ) 117 documentos $(1,47 \%)$ y 3.074 citas; University of Illinois System (UILLINOIS) 108 documentos (1,36\%) y 1.503 citas; University System of Georgia (USG) 102 documentos $(1,28 \%)$ y 2.094 citas; University of California System (UC System) 101 documentos (1,27\%) y 4.346 citas; Louisiana State University (LSU) 99 documentos (1,24\%) y 2.306 citas (tabla 3).

Tabla 3 - Instituciones con mayor producción.

\begin{tabular}{lcccc}
\hline Instituciones & Artículos & $\%$ & Citas & I $^{*}$ \\
\hline California State University (CSU) & 192 & 2,41 & 7.726 & 40,23 \\
University of North Carolina (UNC) & 162 & 2,04 & 4.517 & 27,88 \\
Ohio State University (OSU) & 144 & 1,81 & 3.082 & 21,40 \\
Loughborough University (LBORO) & 142 & 1,78 & 4.463 & 31,42 \\
University of Queensland (UQ) & 117 & 1,47 & 3.074 & 26,27 \\
University of Texas System (UT System) & 113 & 1,42 & 4.814 & 42,60 \\
University of Illinois System (UILLINOIS) & 108 & 1,36 & 1.503 & 13,91 \\
University System of Georgia (USG) & 102 & 1,28 & 2.094 & 20,52 \\
University of California System (UC System) & 101 & 1,27 & 4.346 & 43,02 \\
Louisiana State University (LSU) & 99 & 1,24 & 2.306 & 23,29 \\
\hline
\end{tabular}

Fuente: datos de WOS. Nota: I* Índice de impacto.

Atendiendo a las instituciones con mayor producción, la mayoría están ubicadas en Estados Unidos (EEUU). Por lo que no es de extrañar que sea el país con mayor cuantía de documentos (2.645 documentos, 33,31\%). Sin embargo, Inglaterra presenta un mayor índice de impacto (19.720 citas en 718 documentos). Los demás países obtienen: España 643 documentos (8.09\%) y 5.204 citas; Brasil 575 documentos (7,24\%) y 1.945 citas; Australia 539 documentos (6,78\%) y 11.049 citas; Canadá 391 documentos (4,92\%) y 7.520 citas; Francia 266 documentos (3,35\%) y 3.519 citas; Turquía 230 documentos (2,89\%) y 1.226 citas; China 216 documentos (2,72\%) y 1.280 citas; Grecia 207 documentos $(2,60 \%)$ y 3.871 citas (tabla 4 ). 
Tabla 4 - Países con mayor producción.

\begin{tabular}{lcccc}
\hline Países & Artículos & $\%$ & Citas & $I^{*}$ \\
\hline Estados Unidos & 2.645 & 33,31 & 58.383 & 22,07 \\
Inglaterra & 718 & 9,04 & 19.720 & 27,46 \\
España & 643 & 8,09 & 5.204 & 8,09 \\
Brasil & 575 & 7,24 & 1.945 & 3,38 \\
Australia & 539 & 6,78 & 11.049 & 20,49 \\
Canadá & 391 & 4,92 & 7.520 & 19,23 \\
Francia & 266 & 3,35 & 3.519 & 13,22 \\
Turquía & 230 & 2,89 & 1.226 & 5,33 \\
China & 216 & 2,72 & 1.280 & 5,92 \\
Grecia & 207 & 2,60 & 3.871 & 18,70 \\
\hline
\end{tabular}

Fuente: datos de WOS. Nota: $I^{\star}=$ Índice de impacto.

Respecto a los autores, se establece Mckenzie, T.L. como el más productivo (61 documentos,, $76 \%$ ) y Ntoumanis, N. el más impactante (4.042 citas en 44 documentos). El resto se sitúa con: Hastie, P.A. 47 documentos (,59\%) y 1.012 citas; Kulinna, P.H. 47 documentos (,59\%) y 814 citas; Kirk, D. 46 documentos (,57\%) y 1.595 citas; Ennis, C.D. 43 documentos $(, 54 \%)$ y 1.171 citas; Chen, A. 42 documentos (,52\%) y 822 citas; Xiang, P. 40 documentos (,5\%) y 879 citas; Silverman, S. 39 documentos (,49\%) y 749 citas; Solmon, M.A. 38 documentos (,47\%) y 946 citas (tabla 5).

Tabla 5 - Autores con mayor producción.

\begin{tabular}{lcccc}
\hline Autores & Artículos & $\%$ & Citas & I $^{*}$ \\
\hline Mckenzie, T.L. & 61 &, 76 & 4.535 & 74,34 \\
Hastie, P.A. & 47 &, 59 & 1.012 & 21,53 \\
Kulinna, P.H. & 47 &, 59 & 814 & 17,31 \\
Kirk, D. & 46 &, 57 & 1.595 & 34,67 \\
Ntoumanis, N. & 44 &, 55 & 4.042 & 91,86 \\
Ennis, C.D. & 43 &, 54 & 1.171 & 27,23 \\
Chen, A. & 42 &, 52 & 822 & 19,57 \\
Xiang, P. & 40 &, 5 & 879 & 21,97 \\
Silverman, S. & 39 &, 49 & 749 & 19,20 \\
Solmon, M.A. & 38 &, 47 & 946 & 24,89 \\
\hline
\end{tabular}

Fuente: datos de WOS. Nota: $I^{\star}=$ Índice de impacto.

Por último, siguiendo las consideraciones de Gutiérrez et al. (2015), se ha establecido la relación entre los autores en base a la autoría conjunta de los artículos (figura 6). La relación de los autores con mayor producción se define en dos clúster diferenciados y tres unidades:

- Clúster 1: Chen, A. (centralidad 16); Ennis, C.D. (centralidad 16); Xiang, P. (centralidad 5) y; Solmon, M.A. (centralidad 5).

- Clúster 2: Kirk, D. (centralidad 1); Kulinna, P.H. (centralidad 9) y; Silverman, S. (centralidad 8).

- Unidad 1: Mckenzie, T.L. (centralidad 0).

- Unidad 2: Hastie, P.A. (centralidad 0).

- Unidad 3: Ntoumanis, N. (centralidad 0). 
Figura 6 - Relación entre los autores con mayor producción.

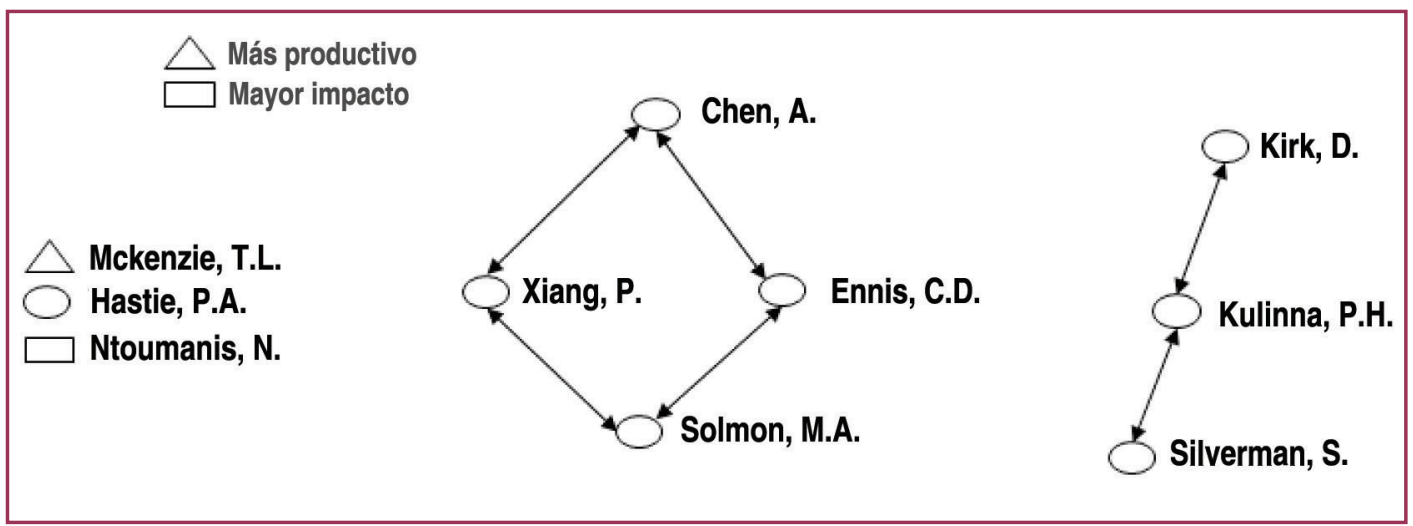

Fuente: elaboración propia a partir de UCINET.

\section{DISCUSIÓN}

Los datos recogidos muestran el avance de la producción científica sobre educación física y su consolidación (VALENCIANO; DEVÍS; BELTRÁN, 2008). Además, la búsqueda de artículos con índice de impacto JCR es un criterio de calidad científica (BUELA-CASAL; ZYCH, 2010; HERNÁNDEZ et al., 2013). Así pues, se han analizado los índices que siguen otros estudios bibliométricos (REVERTER; MUNGUÍA, 2007; VILLAMÓN et al., 2012; REVERTER et al., 2013; OLIVERA; ANDRÉS, 2017; RUBILAR; PÉREZ, 2018; GOMES et al., 2018), como el total de citas, cuantía de trabajos y factor de impacto de revistas, instituciones y autores. Atendiendo, del mismo modo, a la verificación de las leyes de la bibliometría de Price, Lotka y Bradford.

En cuanto a la primera, la productividad diacrónica establece que la literatura se sitúa en plena etapa de crecimiento exponencial, al igual que el análisis bibliométrico sobre evaluación de la educación física escolar durante 1932-2004 (SANTOS et al., 2018). Es de interés resaltar que en el periodo temporal entre 1909-1929 se registra la ausencia de publicaciones o una cuantía mínima. Se constata la verificación de la ley de Price, puesto que se muestra la duplicidad de la producción científica en la mayor parte de periodos temporales quinquenales (PRICE, 1986; GUTIÉRREZ et al., 2015).

En relación a la segunda, se confirma que hay un pequeño grupo de autores que son muy productivos, coincidiendo con lo establecido en la ley de Lotka (ROUSSEAU; ROUSSEAU, 2000). No obstante, la mayor parte de la literatura científica se sustenta en autores que son medianos productores, los cuales poseen entre uno y nueve documentos.

Respecto a la tercera, la ley de Bradford muestra la dispersión de la producción sobre educación física. Si atendemos a los resultados, se verifica claramente esta ley bibliométrica (URBIZAGASTEGUI, 2016). En este sentido, cuatro revistas (núcleo de Bradford) presentan una cuantía mayor de documentos que 755 revistas (zona 4) (ver figura 4).

Por otro lado, las revistas indexadas en JCR donde más publican los docentes españoles y brasileños en Reverter et al. (2014), no coinciden con las 10 primeras revistas que contienen mayor cuantía de documentos en el estudio realizado. En concreto, el núcleo de revistas con mayor cuantía lo compone JTPE, Sport, Education and Society, Research Quarterly for Exercise and Sport (RQES) y Movimento, la cual se consolida como la primera revista brasileña con mayor producción en el escenario internacional. 
Estas revistas se caracterizan por publicar cuatro números al año, excepto Sport, Education and Society que recoge ocho números/año. La temática general se centra en la actividad física y educación física escolar en la mayor parte de ellas y el idioma principal de publicación es el inglés. A diferencia del resto, Movimento también publica manuscritos en portugués y español.

En otra línea, las instituciones estadounidenses se consolidan como las más productoras, ya que recogen una cuantía superior de documentos sobre educación física. Entre las 10 primeras, ocho están situadas en los EEUU. Asimismo, EEUU es el país con mayor producción, seguido de Inglaterra, España y Brasil.

En última instancia, se recoge la relación entre los autores con mayor producción, donde se establecen dos clúster de autorías compartidas. Aunque el autor más productivo (Mckenzie, T.L) y el más impactante (Ntoumanis, N.) no comparten autoría entre los 10 mayores productores.

\section{CONCLUSIONES}

La investigación sobre educación física presenta perspectivas de crecimiento en los próximos años. La consolidación del tópico como eje central de algunas revistas, ha posibilitado que se establezcan un pequeño número de revistas (núcleo de Bradford) como referentes en la temática.

El estudio bibliométrico recoge datos de interés sobre la investigación de impacto de la educación física, mostrando las tendencias que se han ido fijando desde sus inicios hasta el año 2017.

En cuanto a las preguntas de investigación planteadas, se ha dado respuesta a cada una de ellas. De modo que se especifica el estado de la producción científica a lo largo del tiempo, se confirma la relación productiva entre un pequeño grupo de autores y el número de artículos publicados, se verifica la concentración de la mayor parte de artículos en un número reducido de revistas y se han establecido las principales revistas, instituciones, autores y países con mayor producción científica sobre educación física.

Por lo que en suma, se ha atendido el objetivo de investigación planteado acerca de analizar la producción científica sobre educación física, indexada en WOS, desde sus inicios hasta el año 2017.

Por último, remarcar, como prospectiva y líneas futuras de investigación el análisis pormenorizado de los tópicos que recogen las revistas con mayor número de documentos sobre educación física. Es de interés seguir avanzando en futuros trabajos en el análisis bibliométrico de la producción científica, señalando la tendencia por años en las temáticas de las publicaciones acerca de la educación física escolar.

\section{REFERENCIAS}

BUELA-CASAL, Gualberto; ZYCH, Izabela. Analysis of the relationship between the number of citations and the quality evaluated by experts in Psychology journals. Psicothema, v. 22, n. 2, p. 270-276, may 2010. 
GARCÍA, Alfonso Javier. Beneficios de la actividad física sobre la autoestima y la calidad de vida de personas mayores. Retos, n. 33, p. 3-9, 2018.

GLANZEL, Wolfgang; SCHOEPFLIN, Urs. A bibliometric study of reference literature in the sciences and social sciences. Information processing \& management, v. 35, n. 1, p. 31-44, ene. 1999.

GOMES, Iván; QUINTÃO, Felipe; MARINOTTE, Arielle; SAMPAIO, Amanda; ROSSINI, Sergio. O corpo como tema da produção do conhecimento: uma análise bibliométrica em cinco periódicos da educação física brasileira. Movimento, v. 24, n. 2, p. 427-440, abr.jun. 2018. DOI:10.22456/1982-8918.73701

GONZÁLEZ, Javier; MOYA, Manuel; MATEOS, M.A. Indicadores bibliométricos: características y limitaciones en el análisis de la actividad científica. Anales Españoles de Pediatría, n. 47, p. 235-244, 1997.

GUTIÉRREZ, Calixto; MARTíN, Ana; CASASEMPERE, Antoni; FERNÁNDEZ, Antonio. Análisis Cientimétrico de la Grounded Theory en Educación. Revista de Educación, n. 370, p. 121-148, 2015.

HERNÁNDEZ, Vicenç; REVERTER, Joaquín; JOVÉ, María del Carmen; MAYOLAS, María del Carmen. Hábitos de publicación de profesores de los departamentos de didáctica de la expresión corporal y educación física de las universidades públicas españolas. European Journal of Human Movement, n. 30, p. 1-12, 2013.

HINOJO-LUCENA, María Angustias; AZNAR, Inmaculada; CÁCERES, María Pilar. Análisis de las competencias profesionales de la educación física en la etapa de educación primaria. TRANCES, v. 8, n. 1, p. 367-376, 2016.

LÓPEZ, Juan. Educación Física y deporte escolar. Retos, n. 9, p. 19-22, 2006.

MANZANO, Samuel; LÓPEZ, Sebastián; SUÁREZ, Sara; RUIZ, Alberto. Análisis descriptivo y de relación entre la actividad física y el rendimiento académico en jóvenes estudiantes del centro de España. INFAD Revista de Psicología, n. 1, p. 371-376, 2018.

MORAL, Luis. Nivel de actividad física cardiosaludable en educación física en educación primaria: expectativas y algunas evidencias. Sportis, v. 4, n. 1, p. 95-110, 2018. DOI:10.17979/ sportis.2018.4.1.2017

OLIVERA, Javier; ANDRÉS, Ana. Apunts. Educación Física y Deportes (1985-2015). Treinta años en el contexto de las revistas españolas de ciencias de la actividad física y el deporte. Apunts. Educación Física y Deportes, n. 128, p. 9-35, abr./jun. 2017. DOI:10.5672/ apunts.2014-0983.es.(2017/2).128.01

PRICE, Derek J. De Solla. Little Science, big science....and beyond. Nueva York: Columbia University Press, 1986.

RAMÍREZ, María Belén; RAYA, Manuel; RUIZ, Marta. Sedentarismo y salud: efectos beneficiosos de la actividad física en estudiantes universitarios. ReiDoCrea, n. 7, p. 79-84, 2018.

REVERTER, Joaquín; MUNGUÍA, Diego. Estudio comparativo de tres revistas españolas de Educación Física y Deportes. Apunts Educación Física y Deportes, n. 89, p. 102-109, jul./sep. 2007. 
REVERTER, Joaquín; HERNÁNDEZ, Vicenç; JOVÉ, María del Carmen; LEGAZ, Alejandro. Indicadores de producción de los profesores de Educación Física y Didáctica de la Expresión Corporal en España en la Web of Science. Perspectivas em Ciência da Informação, v. 18, n. 3, p. 3-23, 2013.

REVERTER, Joaquín; HERNÁNDEZ, Vicenç; JOVÉ, María del Carmen; FONSECA, Teresa; LEGAZ, Alejandro. Producción de artículos en la base de datos Web of Science y Scopus sobre educación física: estudio comparativo entre España y Brasil. TransInformação, v. 26, n. 2, p. 113-124, may/ago. 2014. DOI:10.1590/0103-37862014000200001

ROUSSEAU, Brendan; ROUSSEAU, Ronald. LOTKA: A program to fit a power law distribution to observed frequency data. Cybermetrics, v. 4, n. 1, p. 1-6, 2000.

RUBILAR, Cristian Alexis; PÉREZ, Mikel. Análisis histórico-bibliométrico de los artículos publicados en revistas científicas chilenas de Ciencias del Deporte durante el gobierno militar (1973-1990). Revista Brasileira de Ciências do Esporte, v. 40, n. 1, p. 46-53, 2018. DOI:10.1016/j.rbce.2018.01.001

SANTOS, Wagner; LIMA, Matheus; CASSANI, Juliana Martins; FERREIRA, Amarílio. Avaliação em educação física escolar: trajetória da produção acadêmica em periódicos (1932-2014). Movimento, v. 24, n. 1, p. 9-22, ene./mar. 2018. DOI:10.22456/1982-8918.63067

URBIZAGASTEGUI, Rubén. El crecimiento de la literatura sobre la ley de Bradford. Investigación bibliotecológica, v. 30, n. 68, p. 51-72, 2016.

VALENCIANO, Javier; DEVÍS, José; BELTRÁN, Vicente Javier. La investigación y las revistas científicas de la actividad física y el deporte en España. Revista Fuentes, n. 8, p. 209-218, 2008.

VILLAMÓN, Miguel; JOB, Ivone; VALCÁRCEL, Javier Valenciano; DEVIS-DEVIS, José. Comparative study on five sport science journals indexed in WoS. Revista de Psicología del Deporte, v. 21, n.2, p.281-287, 2012. 